\title{
A GEOMETRIC INEQUALITY FOR PLANE CURVES WITH RESTRICTED CURVATURE
}

\author{
G. D. CHAKERIAN, H. H. JOHNSON AND A. VOGT
}

\begin{abstract}
A geometric proof is given that a closed plane curve of length $L$ and curvature bounded by $K$ can be contained inside a circle of radius $L / 4-(\pi-2) / 2 K$.
\end{abstract}

Let $K$ be a positive constant and let $E_{n}$ be $n$-dimensional Euclidean space. A continuously differentiable curve $X$ in $E_{n}$ parametrized by arc length $s$ is called a $K$-curve if and only if $\left\|X^{\prime}\left(s_{1}\right)-X^{\prime}\left(s_{2}\right)\right\| \leqslant K\left|s_{1}-s_{2}\right|$ for all $s_{1}$ and $s_{2}$. The purpose of this note is to give a geometric proof of an inequality obtained previously by calculus of variations methods [4]: namely, that if $X$ is a closed $K$-curve in $E_{2}$ of length $L$, then $X$ lies in a circle of radius $R$ where

$$
R \leqslant L / 4-(\pi-2) / 2 K .
$$

Since the components of $X^{\prime}$ are functions of bounded variation, $X^{\prime \prime}(s)$ and $k(s)=\left\|X^{\prime \prime}(s)\right\|$ exist for almost all $s$ and, when $k(s)$ exists, $k(s) \leqslant K$. Thus, $K$ curves are a generalization of $C^{2}$ curves with curvature bounded by $K$. They share many of the geometrical properties of the latter but are to be preferred in several respects. Dubins [3] showed that among $K$-curves with prescribed initial and terminal points and prescribed initial and terminal tangent vectors there exists a $K$-curve of minimal length. We show below (Proposition 3) that the convex envelope of a closed $K$-curve in $E_{2}$ is also a closed $K$-curve. Both of these properties fail if $K$-curves are replaced by $C^{2} K$-curves. In fact, Proposition 3 fails if $K$-curves are replaced by piecewise $C^{2} K$-curves.

To prove inequality (1) we first generalize a theorem of Blaschke [1, p. 116] to the case of convex $K$-curves, then apply a geometrical construction to show that (1) holds for convex $K$-curves, and then extend (1) to all $K$-curves by Proposition 3. An alternative geometric proof of (1) may be obtained by combining results of Dubins [3, Proposition 1 and Theorem 1] with Theorem 2 of Johnson [4]. (We are indebted to the referee for alerting us to Dubins' interesting work and to the existence of the alternative proof.) For information on related problems, consult [2]-[4].

Let $C$ be a closed convex $K$-curve in $E_{2}$ with arc length parameter $s$. (The arc length parameter of a closed $K$-curve is understood to assume all real values by periodic extension. Also, a closed $K$-curve in $E_{2}$ is convex if and only

Received by the editors June 11, 1975 and, in revised form, September 22, 1975. AMS (MOS) subject classifications (1970). Primary 53A05, 52A40.

Key words and phrases. Plane curves, geometric inequalities, convexity. 
if it is simple and its inside is a convex set.) Let $P$ be a point of $C$. With no loss of generality we suppose that $C(0)=P=(0,0)$, that $C^{\prime}(0)=(1,0)$, and that $C$ lies above the $x$-axis.

Let $\tau(s)$ be the unique continuous function such that $\tau(0)=0$ and $C^{\prime}(s)$ $=(\cos \tau(s), \sin \tau(s))$ for all $s$. Since $C$ is convex, $\tau$ is a nondecreasing function of $s$. Since $C$ is a $K$-curve,

$$
4 \sin ^{2}\left[\left(\tau\left(s_{1}\right)-\tau\left(s_{2}\right)\right) / 2\right]=\left\|C^{\prime}\left(s_{1}\right)-C^{\prime}\left(s_{2}\right)\right\|^{2} \leqslant K^{2}\left|s_{1}-s_{2}\right|^{2}
$$

for all $s_{1}$ and $s_{2}$. With $\varepsilon>0$ fixed, it follows that $\left|\tau\left(s_{1}\right)-\tau\left(s_{2}\right)\right| \leqslant(K+\varepsilon)$ $\cdot\left|s_{1}-s_{2}\right|$ for $\left|s_{1}-s_{2}\right|$ sufficiently small. By the triangle inequality the restriction on $s_{1}$ and $s_{2}$ can be removed and, since $\varepsilon$ is arbitrary, the inequality $\left|\tau\left(s_{1}\right)-\tau\left(s_{2}\right)\right| \leqslant K\left|s_{1}-s_{2}\right|$ holds for all $s_{1}$ and $s_{2}$.

We also have that

$$
C(s)=\left(\int_{0}^{s} \cos \tau(t) d t, \int_{0}^{s} \sin \tau(t) d t\right)
$$

and

$$
N(s)=(\sin \tau(s),-\cos \tau(s))
$$

where $N(s)$ is the outer-directed normal to $C$. Thus, the support function $h(s)$, representing the perpendicular distance from $C(0)$ to the tangent line through $C(s)$, is given by

$$
h(s)=N(s) \cdot C(s)=\int_{0}^{s} \sin (\tau(s)-\tau(t)) d t
$$

(cf. [1]).

Let $\Gamma$ be a circle of radius $1 / K$ tangent to $C$ at $P$ and lying on the same side of the tangent line as $C$. If the circle $\Gamma$ is parametrized by arc length $\sigma$ with $\Gamma(0)=P=(0,0)$ and $\Gamma^{\prime}(0)=(1,0)$, then $\Gamma^{\prime}(\sigma)=(\cos K \sigma, \sin K \sigma)$ for all $\sigma$, $\Gamma$ has a support function $h_{0}(\sigma)$ analogous to that of $C$, and $h_{0}(\sigma)=$ $(1-\cos K \sigma) / K$ by an elementary computation.

Suppose $\Gamma$ crosses $C$. By the Mean Value Theorem there will exist points $\Gamma(\sigma)$ and $C(s)$ where the tangent vectors $\Gamma^{\prime}(\sigma)$ and $C^{\prime}(s)$ are the same and $h_{0}(\sigma)$ is larger than $h(s)$. The parameter values $\sigma$ and $s$ may be chosen to satisfy $K \sigma=\tau(s)$ and without loss of generality we may assume that $0<K \sigma=\tau(s)$ $\leqslant \pi$. Then $\sigma$ and $s$ are positive and

$$
\begin{aligned}
h_{0}(\sigma) & =\frac{1}{K} \int_{0}^{\tau(s)} \sin (\tau(s)-\tau) d \tau \\
& =\lim _{\operatorname{mesh} \rightarrow 0} \sum_{i=1}^{n} \sin \left(\tau(s)-\tau\left(s_{i}\right)\right)\left(\tau\left(s_{i}\right)-\tau\left(s_{i-1}\right)\right) / K \\
& \leqslant \lim _{\operatorname{mesh} \rightarrow 0} \sum_{i=1}^{n} \sin \left(\tau(s)-\tau\left(s_{i}\right)\right)\left(s_{i}-s_{i-1}\right) \\
& =\int_{0}^{s} \sin (\tau(s)-\tau(t)) d t=h(s) .
\end{aligned}
$$

Here $s_{0} \leqslant \cdots \leqslant s_{n}$ is a partition of $[0, s]$ and $\tau\left(s_{0}\right) \leqslant \cdots \leqslant \tau\left(s_{n}\right)$ is the 
corresponding partition of $[0, \tau(s)]$. Our conclusion is that $h_{0}(\sigma) \leqslant h(s)$ contrary to hypothesis, and so we have proved

Proposition 1 (BlaschKe). Let $C$ be a closed convex $K$-curve in $E_{2}$. Then through each point $P$ of $C$ passes a circle $\Gamma$ of radius $1 / K$ tangent to $C$ at $P$ and lying inside $C$.

Now, if $C$ has length $L$, let $A=C\left(s_{1}\right)$ and $B=C\left(s_{1}+L / 2\right)$ be two points where $C^{\prime}\left(s_{1}\right)=-C^{\prime}\left(s_{1}+L / 2\right)$. Such points exist by a continuity argument. Let $M$ be the midpoint of the line segment $A B$ and let $P$ be an arbitrary point of $C$.

$A$ and $B$ divide $C$ into two arcs, one of which contains $P$. Reflect the arc containing $P$ centrally through $M$ to obtain a new curve $C^{*}$ (see Figure 1) of the same length $L$ but now symmetric about $M$.

Like $C, C^{*}$ is a closed convex $K$-curve. Hence, by Proposition 1 , there is a circle $\Gamma$ of radius $1 / K$ tangent to $C^{*}$ at $P$ and lying inside $C^{*}$. Its central reflection $\Gamma_{0}$ through $M$ also lies inside $C^{*}$. Likewise, the convex envelope $\Gamma^{*}$ of $\Gamma \cup \Gamma_{0}$ lies inside $C^{*}$. Let $P_{0}$ be the center of $\Gamma$. Then the length of $\Gamma^{*}$ is $2 \pi / K+4 d\left(M, P_{0}\right)$ and is less than or equal to $L$. But

$$
d(M, P) \leqslant d\left(M, P_{0}\right)+d\left(P_{0}, P\right)=d\left(M, P_{0}\right)+1 / K .
$$

Hence, $d(M, P) \leqslant L / 4-(\pi-2) / 2 K$. Since $P$ was an arbitrary point of $C$, we conclude that $C$ lies inside a circle of radius $\leqslant L / 4-(\pi-2) / 2 K$ centered at $M$.

If $d(M, P)=L / 4-(\pi-2) / 2 K$ for some point $P$ as above, then the inequalities become equalities and it follows that $M, P_{0}$, and $P$ are collinear in that order and $\Gamma^{*}=C^{*}$. In such a case $C^{*}$ is a "racetrack" curve, that is, $C^{*}$ is the convex envelope of two circles of radius $1 / K$ whose centers are a distance $L / 2-\pi / K$ apart. The $\operatorname{arc} A P B$ of the original curve $C$ is half of $C^{*}$ and thus is half of a racetrack curve. If a point $Q$ on the arc of $C$ opposite to $A P B$ can be found with $d(M, Q)=L / 4-(\pi-2) / 2 K$, then $A Q B$ is also half of a racetrack curve and $C=A P B \cup A Q B$ is a racetrack curve. If no such point $Q$ can be found, then $d(M, A)=d(M, B)<L / 4-(\pi-2) / 2 K$ and the center $M$ can be shifted toward $P$ so as to obtain a circle of radius $<L / 4-(\pi-2) / 2 K$ containing $C$.

We have proved

Proposition 2. Let $C$ be a closed convex $K$-curve in $E_{2}$ of length $L$. Then $C$ lies inside a circle of radius $L / 4-(\pi-2) / 2 K$. The curve $C$ may be contained in no smaller circle precisely when $C$ is a racetrack curve of length $L$ and radii $1 / K$.

Let $X$ be a closed $K$-curve and let $C$ be the convex envelope of $X$. Observe that every point of $C$ is either a point of $X$ or else an interior point of a line segment in $C$ whose endpoints lie on $X$. At the latter points, of course, $C$ has its tangent line parallel to the line segment. At points of $C \cap X$ there is likewise a unique supporting line of $C$ : for, if the supporting lines formed a cone at such a point, $X$ could not have a derivative there.

Let $\sigma$ be an arc length parameter for $X$ and let $s$ be an arc length parameter 
for $C$. By what has just been said, $C^{\prime}(s)$ is a well-defined tangent vector and at points of $C \cap X$ coincides up to sign with $X^{\prime}(\sigma)$.

Let $\varepsilon$ be a positive number and $s_{0}$ a particular value of $s$. We shall show that for $s$ sufficiently close to $s_{0}$,

$$
\left\|C^{\prime}(s)-C^{\prime}\left(s_{0}\right)\right\| \leqslant(K+\varepsilon)\left|s-s_{0}\right| .
$$

Suppose to the contrary that there exists a sequence $\left\{s_{n}\right\}$ convergent to $s_{0}$ such that $\left\|C^{\prime}\left(s_{n}\right)=C^{\prime}\left(s_{0}\right)\right\|>(K+\varepsilon)\left|s_{n}-s_{0}\right|$ for all $n$. If $C\left(s_{0}\right)$ is not on $X$, then for $n$ large $C\left(s_{n}\right)$ is on the line segment of $C$ through $C\left(s_{0}\right)$. Thence, $C^{\prime}\left(s_{n}\right)$ equals $C^{\prime}\left(s_{0}\right)$ for a contradiction. So, $C\left(s_{0}\right)$ must belong to $C \cap X$. We can suppose too that, for each $n, C\left(s_{n}\right)$ belongs to $C \cap X$. Otherwise, the points $C\left(s_{n}\right)$ would be interior points of line segments of $C$. Without affecting $C^{\prime}\left(s_{n}\right)$ or increasing $\left|s_{n}-s_{0}\right|$, we could then shift the points along the segments until they met $X$.

The curve $X$ has only a finite number of branches which go through $C\left(s_{0}\right)$. By passing to a subsequence, we can suppose that the points $C\left(s_{n}\right)$ all lie on the same branch of $X$. Thus, there exists a sequence $\left\{\sigma_{n}\right\}$ converging to a parameter value $\sigma_{0}$ with $X\left(\sigma_{0}\right)=C\left(s_{0}\right)$ and $X\left(\sigma_{n}\right)=C\left(s_{n}\right)$ for all $n$. By passing to subsequences once more, we can assume that, for all $n, C^{\prime}\left(s_{n}\right)$ $=u X^{\prime}\left(\sigma_{n}\right)$ where $u= \pm 1$ is fixed. But $\left\{X^{\prime}\left(\sigma_{n}\right)\right\}$ converges to $X^{\prime}\left(\sigma_{0}\right)$. Hence, $\left\{C^{\prime}\left(s_{n}\right)\right\}$ converges to a limit which up to sign equals $X^{\prime}\left(\sigma_{0}\right)$ and, therefore, up to sign equals $C^{\prime}\left(s_{0}\right)$. Since $C$ is a closed convex curve, it cannot reverse direction abruptly. So $\left\{C^{\prime}\left(s_{n}\right)\right\}$ converges to $C^{\prime}\left(s_{0}\right)$ and $C^{\prime}\left(s_{0}\right)=u X^{\prime}\left(\sigma_{0}\right)$.

Then,

$$
\begin{aligned}
(K+\varepsilon)\left|s_{n}-s_{0}\right| & <\left\|C^{\prime}\left(s_{n}\right)-C^{\prime}\left(s_{0}\right)\right\|=\left\|u\left(X^{\prime}\left(\sigma_{n}\right)-X^{\prime}\left(\sigma_{0}\right)\right)\right\| \\
& =\left\|X^{\prime}\left(\sigma_{n}\right)-X^{\prime}\left(\sigma_{0}\right)\right\| \leqslant K\left|\sigma_{n}-\sigma_{0}\right| .
\end{aligned}
$$

But for any positive number $\alpha<1$ and for $n$ sufficiently large,

$$
\left\|\left(X\left(\sigma_{n}\right)-X\left(\sigma_{0}\right)\right) /\left(\sigma_{n}-\sigma_{0}\right)\right\|>1-\alpha .
$$

Hence,

$$
\begin{aligned}
(K+\varepsilon)\left|s_{n}-s_{0}\right| & <K\left|\sigma_{n}-\sigma_{0}\right|<K\left\|X\left(\sigma_{n}\right)-X\left(\sigma_{0}\right)\right\| /(1-\alpha) \\
& =K\left\|C\left(s_{n}\right)-C\left(s_{0}\right)\right\| / 1-\alpha \leqslant K\left|s_{n}-s_{0}\right| /(1-\alpha)
\end{aligned}
$$

for such $n$. Thus, $K+\varepsilon<K /(1-\alpha)$ and, letting $\alpha$ vary, we conclude that $K+\varepsilon \leqslant K$ for a contradiction.

We have established that for each value $s_{0}$ and for each $s$ sufficiently close to $s_{0},\left\|C^{\prime}(s)-C^{\prime}\left(s_{0}\right)\right\| \leqslant(K+\varepsilon)\left|s-s_{0}\right|$. By a compactness argument plus the triangle inequality, it follows that $C$ is a $(K+\varepsilon)$-curve. Since this is true for an arbitrary positive number $\varepsilon, C$ is a $K$-curve.

Proposition 3. Let $X$ be a closed $K$-curve in $E_{2}$. Then the convex envelope $C$ of $X$ is a closed convex $K$-curve.

Since the length of $C$ is at most that of $X$, we have our main result by combining Propositions 2 and 3. 
Theorem 1. Let $X$ be a closed $K$-curve in $E_{2}$ of length $L$. Then $X$ lies inside a circle of radius $L / 4-(\pi-2) / 2 K$. The curve $X$ may be contained in no smaller circle precisely when $X$ is a racetrack curve of length $L$ and radii $1 / K$.

\section{REFERENCES}

1. W. Blaschke, Kreis und Kugel, Viet, Leipzig, 1916; reprint, Chelsea, New York, 1949. MR $17,887$.

2. G. D. Chakerian and M. S. Klamkin, Minimal covers for closed curves, Math. Mag. 46 (1973), 55-61. MR 47 \#2496.

3. L. E. Dubins, On curves of minimal length with a constraint on average curvature, and with prescribed initial and terminal positions and tangents, Amer. J. Math. 79 (1957), 497-516. MR 19, 678.

4. H. H. Johnson, An application of the maximum principle to the geometry of plane curves, Proc. Amer. Math. Soc. 44 (1974), 432-435. MR 50 \#1128.

Department of Mathematics, University of California, Davis, California 95616 (Current address of G. D. Chakerian)

Department of Mathematics, University of Washington, Seattle, Washington 98195

Department of Mathematics, Georgetown University, Washington, D. C. 20057 (Current address of A. Vogt)

Current address (H. H. Johnson): 10900 Hunt Club Road, Reston, Virginia 22090 